\title{
Una explicación del abstencionismo electoral en la primera vuelta de las Elecciones Presidenciales de 2014 en Colombia $^{1}$
}

\section{An explanation of electoral abstention in the first round of the 2014 presidential elections in Colombia}

\section{John Fredy Bedoya Marulanda iD}

Universidad de Antioquia

john.bedoyam@udea.edu.co

\section{Felipe Nieto Palacio}

Universidad de Antioquia

felipe.nieto@udea.edu.co

\begin{abstract}
Resumen
Si bien la participación electoral ha sido ampliamente estudiada desde la ciencia política, el abstencionismo no ha recibido el mismo énfasis empírico como fenómeno característico en el comportamiento ciudadano. En tal sentido, el presente trabajo busca identificar las razones que explican la ausencia de los electores en las urnas, asumiendo como caso de estudio la primera vuelta de las Elecciones Presidenciales de 2014 en Colombia. Estos comicios son analíticamente importantes por el alto porcentaje de abstencionismo registrado. Con un potencial electoral de 33'266.272, se depositaron 13'222.354 votos, lo que corresponde a un abstencionismo de 60,25\%, uno de los porcentajes más altos en la historia reciente de las elecciones en Colombia. Para lograr el objetivo de explicar el abstencionismo, se toma como unidad de análisis todos los municipios del país, con el fin de realizar un análisis agregado de los datos. Además, se recurre a los resultados de la encuesta del Barómetro de las Américas-Lapop de 2016 para analizar a nivel individual los determinantes de la abstención.
\end{abstract}

Palabras clave: Abstencionismo, elecciones, democracia, participación electoral, comportamiento electoral.

\begin{abstract}
Although voter turnout has been widely studied in political science, abstention has not received the same empirical emphasis as a characteristic phenomenon in citizen behavior. In this regard, this paper seeks to identify the reasons that explain the absence of voters at the polls, assuming as a case study the first round of the 2014 presidential elections in Colombia. These elections are analytically important because of the high percentage of abstention recorded. With an electoral potential of 33.266.272, 13.222.354 votes were deposited, which corresponds to an abstention of 60.25\%, one of the highest percentages in the recent history of the elections in Colombia. To achieve the objective, all the municipalities
\end{abstract}

${ }^{1}$ Este artículo es producto de la investigación "Electores urbanos y rurales: una misma competencia, intereses diferenciados. Elecciones para Alcalde y Presidente, 2003-2014". 
in the country are taken as the unit of analysis, in order to implement an aggregate analysis of the data. In addition, we use the results of the 2016 Americas Barometer- LAPOP survey to analyze the determinants of abstention at the individual level.

Keywords: Abstention, elections, democracy, voter turnout, electoral behavior.

Articulo: Recibido el 4 de marzo de 2019 y aprobado el 15 de octubre de 2019.

\section{Cómo citar este artículo:}

Bedoya Marulanda, J.F. \& Nieto Palacio F. (2020). Una explicación del abstencionismo electoral en la primera vuelta de las Elecciones Presidenciales de 2014 en Colombia. Reflexión Política 22(44), pp. 9-25. doi: 10.29375/01240781.3359

\section{Introducción}

Durante las elecciones los individuos tienen dos alternativas: pueden ejercer su derecho al sufragio como respuesta legitimadora del sistema político o, desencantados por el accionar de los gobernantes y de los partidos políticos, pueden abstenerse aun afrontando distintas penalidades. En cierta medida, esta decisión podría entenderse haciendo un símil con los postulados de Albert Hirschman (1977), para quien el voto es la voz de los ciudadanos que permite transformar las prácticas y políticas del organismo al que pertenecen, mientras que la abstención es la salida del sistema, por la cual optan los individuos ante un bajón en la calidad de la oferta política.

Frente a este asunto, la literatura política se ha ocupado mayoritariamente de entender la participación electoral, cuyas explicaciones han evidenciado la complejidad que hay detrás de la decisión de asistir a las urnas, mostrando el entramado de factores sociales, políticos, institucionales, culturales e individuales que hay detrás de esta (Geys, 2006). Sin embargo, el abstencionismo denota una mayor dificultad en su análisis, no solo por su carácter multifactorial, sino porque es necesario analizar una parte del electorado que no expresa sus preferencias.

Así, una de las maneras en la que se ha entendido el abstencionismo es la no participación en un acto al cual se tiene derecho (Alcubilla, 2002). Visto así, como un no hecho, ha sido abordado principalmente desde el plano prescriptivo, pues la no participación electoral de los ciudadanos parece un síntoma del mal funcionamiento de los procesos democráticos: si la participación se vincula con el empoderamiento del pueblo, justificador del poder constituido, la abstención da muestra de problemas de legitimidad. De esta manera, el abstencionismo pone en vilo los principios de la democracia representativa puesto que la ausencia de electores en las urnas configura una difusa y parcial relación entre representantes y representados, en tanto los dirigentes escogidos popularmente encarnan los valores y principios de solo algunos votantes, lo que podría traducirse en el desconocimiento e invisibilización de los intereses del resto de la ciudadanía.

Por otro lado, cuando la abstención se entiende como la decisión consciente de optar por la salida del proceso electoral, los estudios han señalado que sus variaciones al margen pueden explicarse por variables que dan cuenta del capital individual de los ciudadanos, como ingresos o niveles de estudio; variables sociales como las redes y la comunicación; factores institucionales como los incentivos que ofrece el sistema político, así como las dinámicas del sistema de partidos; y variables psicológicas que dan cuenta de las actitudes que expresan los individuos hacia el sistema político en su conjunto.

Para el caso colombiano en particular, aun cuando el abstencionismo ha sido una constante al momento de referenciar las dinámicas del ejercicio electoral, su abordaje se hace mayoritariamente desde el plano normativo y enunciativo, aduciendo a fenómenos como el malestar de la ciudadanía hacia los procesos electorales, los cuales han estado impregnados de males como la corrupción y el clientelismo (Uribe, 1996). Es por ello que se hacen necesarios estudios que se preocupen por explicar 
la variabilidad del abstencionismo y sus matices ${ }^{2}$.

En ese sentido, este artículo se pregunta por los factores que explican el abstencionismo electoral en Colombia. Con el fin de responder a esta pregunta, se usa como caso de estudio la primera vuelta de las elecciones presidenciales de 2014, desde dos frentes diferentes. En el primero se toman como unidad de análisis a todos los municipios del país con el fin de determinar, mediante una regresión lineal múltiple, la relación entre la abstención y algunas condiciones sociodemográficas como el tamaño de la población, la capacidad económica y la violencia; factores relevantes en la literatura sobre el tema. En el segundo frente, se recurre a los resultados de la encuesta del Barómetro de las Américas-Latin American Public Opinion Project en su versión de 2016 para identificar, a través de una regresión logística, la incidencia de factores actitudinales individuales que pueden explicar la decisión de abstenerse.

Este trabajo adquiere relevancia dada la precaria literatura alrededor de fenómenos electorales en América Latina, y en especial sobre abstencionismo, por lo que responde al llamado a desarrollar con mayor insistencia tal subcampo de estudio (Bedoya, 2015). Además, el uso de las elecciones del 2014 es importante analíticamente; en primer lugar, por el alto porcentaje de abstención registrado, con un potencial electoral de 33'266.272, se depositaron 13'222.354 votos, lo que corresponde a un abstencionismo del 60,25 \%, uno de los porcentajes más altos en la historia reciente de las elecciones en Colombia. En segundo lugar, por el polarizado contexto político en el cual se inscribía el país, determinado por las negociaciones con la guerrilla de las FARC. Esto produjo que la agenda electoral estuviera permeada por la narrativa de la paz ante un posible acuerdo que diezmara la intensidad de un conflicto que data desde mediados del siglo XX, tema que podría ser crucial para los electores. Otra razón que da relevancia analítica a estas elecciones es que el principal candidato, el presidente Juan Manuel Santos, buscaba su reelección al cargo para el periodo 2014-2018, lo que podría ayudar a probar las hipótesis actitudinales de los votantes, y al que se le sumó la victoria en los comicios legislativos del partido de oposición, liderado por el expresidente
Álvaro Uribe Vélez. Todo esto anticipaba una reñida contienda entre dos posturas distintas de orientar la política de Estado (Caicedo, 2014).

El artículo está estructurado de la siguiente manera: en primer lugar, se exponen los hallazgos que la literatura ha arrojado sobre el abstencionismo y la participación electoral, con el fin de identificar los principales factores que han sido empleados para explicar el objeto de estudio. Acto seguido, se describen los datos electorales de los comicios presidenciales de 2014 para comprender las dinámicas municipales $\mathrm{y}$ regionales del abstencionismo. También se expone el modelo de regresión lineal empleado y los resultados encontrados. Luego se describen los datos individuales y se presenta el modelo de regresión logística, con el que se da cuenta de las explicaciones a nivel micro de la abstención. Finalmente, se plantean las conclusiones del estudio.

\section{1. ¿Qué explica el abstencionismo electoral?}

El estudio de las elecciones ha sido una constante en el desarrollo de la ciencia política. Principalmente desde la perspectiva anglosajona, los análisis sobre el comportamiento electoral han concentrado sus esfuerzos en entender la forma en la que los electores toman sus decisiones, especialmente la de asistir a las urnas. Aunque ha sido sustantiva la producción académica sobre la participación electoral, el abstencionismo no ha recibido el mismo énfasis como fenómeno característico en el comportamiento de los votantes. En términos generales, el abstencionismo ha sido estudiado como un espejo, si se quiere negativo, de la participación electoral. Sin embargo, ante el incipiente desarrollo del objeto de estudio en cuestión, se traen también a colación, con el fin de completar el panorama, las interpretaciones que responden a la pregunta de por qué la gente asiste a votar el día de las elecciones.

\subsection{Una aproximación al estudio de la abstención electoral}

El abstencionismo, en lo que a su motivación refiere, puede manifestar una voluntad de protesta

\footnotetext{
${ }^{2}$ Aquí se hace referencia a las diferencias que presenta el abstencionismo en el país. Por ejemplo, en las elecciones locales, este ha disminuido desde la entrada en vigencia de la Constitución de 1991 y la reforma política de 2003, mientras que en los comicios legislativos, los niveles de abstención en los últimos veinte años oscilan entre el $54 \%$ y $57 \%$. Con respecto a las elecciones presidenciales entre 1978 y 2010 , se promedia una tasa de abstención de $45,99 \%$, destacándose que 10 de las 12 elecciones registran que se han abstenido más personas de las que salieron a votar (Registraduría Nacional del Estado Civil, Centro de Estudios en Democracia y Asuntos Electorales (CEDAE), Escuela de Política y Relaciones Internacionales - Grupo de Análisis Político (GAP), 2013).
} 
ante el sistema político, un síntoma de apatía o incluso un cálculo racional de costos y beneficios. En particular, la abstención ha sido interpretada como un mecanismo que expresa un malestar hacia los procesos políticos que se llevan a cabo en el seno de la democracia. Sin embargo, al ser el abstencionismo un fenómeno complejo, no puede ser entendido de manera unidimensional (Nohlen, 2004). Es por esto que es necesario visibilizar los múltiples factores que configuran el abstencionismo electoral.

En el estudio del comportamiento electoral es recurrente encontrar relaciones entre las características socioeconómicas de los individuos y el acto o no de votar. La teoría de los recursos planteada por Verba y Nie (1972) propone que el nivel económico es determinante para explicar la asistencia a las urnas, pues los ciudadanos con mayores ingresos disponen de más tiempo y de mayores herramientas educativas para informarse sobre la política, lo que los hace menos propensos a abstenerse. Esto es demostrado por Power y Roberts (1995), quienes concluyen que el crecimiento económico está en relación inversa con el abstencionismo, pues el aumento significativo del desarrollo económico disminuye el número de ciudadanos que decide no asistir a las urnas. Millán (2013) encuentra resultados similares al afirmar que la pobreza monetaria incide decisivamente en las altas tasas de abstencionismo presentadas en México.

La escolaridad también ha sido usada como factor explicativo. Bajo el supuesto de que los individuos más educados tienden a estar más involucrados en la política $\mathrm{y}$, por ende, obtienen mayor información que les permite reducir los costos de votación, los estudios de Lutz (2005) para México, Boix y Riba (2000) en España y Medina (2004) en Venezuela explican que los ciudadanos con niveles educativos más bajos se abstienen en mayor proporción que aquellos con niveles de estudios más altos.

Por su parte, Araya (2014), Zavala (2005), Medina (2004) y Hernández (1990) al usar la edad como variable explicativa, coinciden en que mientras más jóvenes sean los ciudadanos, se presentarán tasas más altas de abstención. Esto responde a que en la adolescencia los votantes tienden a tener preferencias menos definidas, lo que los aparta de las dinámicas políticas, hasta que llegan a la adultez, cuando empiezan a recurrir a las urnas más consistentemente.

El tamaño de la población es otro factor que contribuye a entender la abstención. Esta variable se conjuga con el apego de los individuos en comunidad, pues en poblaciones más pequeñas el vínculo entre los ciudadanos es mayor, lo que podría traducirse en mayores flujos de información, de tal manera que el voto se muestra como la expresión del empoderamiento ciudadano que es logrado a través de la socialización recurrente de los ciudadanos. En ese sentido, Boix y Riva (2000) establecen que la vida asociativa de los individuos determina al abstencionismo. Es decir, que la existencia de una menor vida asociativa contribuye a alejar a los ciudadanos de las urnas, pues se abstienen de participar en actividades que podrían dotarlos de información sobre los candidatos y los partidos. Asimismo, estos autores confirman que a mayor tamaño de población la probabilidad de abstenerse es menor, ya que los núcleos grandes conducen a limitar el control social y, por ende, a reducir la resistencia de los ciudadanos para votar.

Por su parte, las variables psicológicas en el estudio de la participación electoral remiten exclusivamente a los procesos mentales que anteceden una decisión electoral. El acto de votar o no, desde este enfoque, se concibe como el resultado de múltiples factores que pueden tener incidencia a corto y largo plazo, incluyendo creencias, valores, actitudes y sentimientos, especialmente frente al sistema político. Desde esta perspectiva, Medina (2004) sugiere que los bajos niveles de participación en Venezuela se explican por la falta de interés en la política. En la misma vía, Zavala (2010), al analizar las elecciones mexicanas, destaca la relación entre el nulo interés en la política y el abstencionismo. Para González (1989), la apatía como condicionante del abstencionismo se expresa como un acto de aceptación pasiva del sistema, en tanto el ciudadano se desentiende de las lógicas electorales. Esto se evidencia en los hallazgos de Correa y Forero (2014), quienes resuelven de manera exploratoria que el abstencionismo en Bogotá responde a la apatía hacia el sistema electoral, los candidatos y las propuestas.

Quizá una de las variables psicológicas que mayor incidencia tiene al momento de explicar el abstencionismo remite a la desafección política. Como lo propone Torcal (2006), la desafección 
política se define como el conjunto de sentimientos de ineficacia, cinismo y falta de confianza en el proceso político y las instituciones democráticas. Mientras más insatisfechos se sientan los ciudadanos con respecto al accionar de los poderes públicos, es más probable que su ausencia en las urnas se entienda como una posición activa de rechazo y descontento al sistema político. Son múltiples los estudios que confirman dicho postulado. Zazueta, Mikhail y Cortéz (2014), Salazar y Temkin (2007), Mijares (2006) y Millán (2013) concuerdan en que el abstencionismo en México podría simbolizar una forma de manifestar inconformidad y desconfianza hacia las instituciones, cuando los electores no se sienten representados. Para el caso colombiano, Giraldo (2012) revela que la desconfianza en las instituciones, la poca relevancia partidista y el sentimiento de exclusión por parte de la ciudadanía son los principales factores que motivaron la abstención en las elecciones locales en Medellín entre 2000 y 2007.

$\mathrm{El}$ orden institucional de las elecciones también ha sido tomado en cuenta para explicar la abstención. Bruno Luzt (2005) asegura que, para los comicios mexicanos de 2003, la naturaleza de la elección es una variable fundamental al momento de comprender la ausencia de los votantes en las urnas. La tasa de abstención es superior cuando se llama a los ciudadanos a elegir a sus representantes en las cámaras de diputados y senados, quizá porque estos no tienen la misma visibilidad política que el presidente ni la cercanía de los gobernadores locales.

En lo concerniente a las configuraciones del sistema electoral, como condicionante del abstencionismo, Riera (2012) y Boix y Riba (2000) arrojan resultados positivos al respecto. En el primer caso, el autor considera que un sistema menos proporcional produce un mayor abstencionismo, puesto que estos sistemas obligan a los partidos políticos pequeños a obtener muchos más votos para lograr un escaño, disminuyendo los beneficios para los seguidores de dichos partidos, desestimando la votación. En el segundo caso, los autores concluyen que el umbral y el tamaño del distrito inciden en las tasas de abstención, pues la reducción del grado de proporcionalidad conduce a la alienación y abstención de aquellos votantes de partidos con pocas probabilidades de elección.

La teoría de la elección racional también ha sido empleada para explicar el abstencionismo electoral. Según Anthony Downs (1957), los individuos actúan a partir de los cálculos racionales que realizan al momento de tomar decisiones políticas. En ese sentido, un elector acude a las urnas cada vez que los beneficios de votar superan los costos de realizar dicho acto. Según lo anterior, un sujeto menos informado tendrá que emplear más recursos para votar, hasta el punto en que para este los costos superen los beneficios, lo que resultaría en abstención.

Otra perspectiva de la teoría de la elección racional usada para explicar la abstención se refiere al modelo espacial del voto. Gershtenson y Plane (2004) y Adams, Dow y Merril (2006) concluyen que hay dos motivos por los cuales ocurre la abstención en elecciones con dos candidatos: una, cuando el ciudadano no encuentra diferencias significativas en las plataformas políticas de los candidatos en contienda; y otra, que sucede cuando el ciudadano siente que ningún candidato representa sus preferencias políticas, por lo que decide abstenerse, en cuanto no encuentra propuestas en su zona de aceptabilidad.

\subsection{Otras variables que pueden explicar la abstención}

Como se mencionó anteriormente, dado que el tema del abstencionismo carece de suficiente desarrollo teórico, resulta necesario tener en consideración los aportes que han sido utilizados para entender la participación electoral, entre estos, los factores institucionales. Con relación al sistema de partidos, Jackman y Miller (1995) sostienen que los sistemas multipartidistas experimentan más abstencionismo en sus comicios electorales. Es decir, que mientras más partidos haya en competencia, menor es el número de votantes que acudirán a las urnas. Una explicación de esta relación se encuentra en Fornos, Power y Garand (2004), quienes afirman que el multipartidismo, al ser compatible con las coaliciones, hace que los votantes sientan menos eficacia en su voto porque perciben que no seleccionan directamente al gobierno. En contravía a lo anterior, Boulding y Brown (2013) afirman que la existencia de menos partidos incentiva la abstención, pues las alternativas en la oferta electoral se reducen $\mathrm{y}$, por lo tanto, hay menos probabilidades de que los votantes se identifiquen con alguna opción. 
Otra variable institucional recurrente es la obligatoriedad del voto, puesto que su existencia tiende a incrementar los costos de no votar debido a la posibilidad de ser sancionado (Fornos et al., 2004; Freitag, 2010). Sumado a lo anterior, la frecuencia con la que los individuos son llamados a sufragar también incide en la participación electoral. Mientras más veces tenga que ir un ciudadano a votar, más proclive será de sufrir de fatiga electoral y se abstendrá en mayor medida (Jackman y Miller, 1995; Boyd, 1986). Esto se relaciona con la simultaneidad de las elecciones, ya que la participación electoral disminuye si los comicios locales y nacionales se realizan en momentos distintos (Caren, 2007; Delgado, 1998; Hajnal y Lewis, 2003; Kuenzi y Lambright, 2007).

También es posible encontrar una relación positiva entre lo reñido de las elecciones y la participación electoral (Breux, Couture y Goodman, 2016): mientras las campañas sean menos competitivas, el interés de los votantes se reducirá restándole así importancia al voto. Otro argumento usado para explicar el fenómeno en cuestión, está asociado con las apuestas electorales o stakes. Eso significa que cuando hay menos en juego en el contexto electoral, los individuos votarán menos (Andersen et al., 2014; Lefevere y Van Aelst, 2014).

El sentido de comunidad, entendido como el apego de los ciudadanos a su territorio y el interés colectivo por los asuntos políticos, sociales y económicos que les competen, constituyen otra fuente explicativa para analizar las variaciones en la participación electoral. Es por esto que el tamaño de la población se relaciona inversamente con la participación, en cuanto funciona como un condicionante del nivel de apego y sentido de la comunidad (Ben-Bassat y Dahan, 2012; Ortega y Trujillo, 2013; Caren, 2007; Frandsen, 2002; Funk, 2008). Este vinculo se fundamenta en el principio de que las comunidades de tamaño reducido tienden a favorecer el imaginario sobre el elector de que no ir a votar es una acción reprochable. Esto significa que las poblaciones más pequeñas tienden a reforzar los dispositivos de control social entre sus ciudadanos, por lo que se hace más visible el comportamiento el día de las elecciones. Aquí también entra en juego la heterogeneidad de la población (Kaniovski y Mueller, 2006; Lago et al., 2014; Belletini, Berti y Monfardini, 2014), pues entre más diverso sea un grupo en términos de su idioma y sus características étnicas, la tasa de asistencia a las urnas será más baja, sobre todo si los ciudadanos tienen un débil sentido de deber cívico.

La comunicación social y política también entran en este grupo de variables. Siguiendo a Downs (1957), se puede percibir que el costo de información y la participación electoral están inversamente relacionados, lo que significa que mientras más esfuerzos deba realizar un elector para obtener información sobre los candidatos y los partidos, hay menores probabilidades de que asista a las urnas el día de las elecciones. De esta manera se identifican tres fuentes según las cuales los votantes pueden adquirir información para acudir a votar: los partidos políticos, los medios de comunicación y el proceso de socialización política. Los partidos funcionan como referentes ideológicos que traducen la información en contextos de campaña con el objetivo de estimular la participación de los ciudadanos. Los medios de comunicación son fundamentales para el proceso electoral, pues, como lo plantea Sørensen (2015), pueden generar impactos sobre el conocimiento alrededor del desempeño de los gobiernos y, por ende, determinar el grado de información que adquieren los individuos. Acorde con lo anterior, se ha encontrado que la exposición a los medios tiende a aumentar la habilidad individual de votar (Kuenzi y Lambright, 2007). La socialización política también es otro medio de transmisión de información, pues el contacto cotidiano en espacios sociales produce intercambio de datos que pueden estar relacionados con los procesos electorales, pero este último factor debe ser leído a la luz del deber cívico y el tamaño de la población.

Cabe resaltar que, a pesar del precario desarrollo de los estudios sobre el comportamiento electoral en Colombia, los pocos aportes enfatizan las particularidades del contexto, como el bipartidismo y la violencia política (Bedoya, 2015). Hoyos (2009), Horbath (2004) y Fernández y Aguilera (2002) son muestra de ello al explicar que la violencia inhibe la participación pública de los ciudadanos y los partidos políticos, lo que se traduce en tasas más bajas de participación electoral.

\section{Distribución geográfica y explicaciones macro de la abstención}

Si bien no existe un parámetro que permita identificar cuándo los niveles de abstención son muy altos o muy bajos, con el objetivo de 
comprender su distribución en la primera vuelta presidencial de 2014, se clasificaron los municipios en cuatro grupos según se muestra en la Tabla $1^{4}$. De acuerdo a esta clasificación, se encuentra a nivel regional que el Caribe, el Pacífico y el Amazonas son las zonas que presentan un mayor porcentaje de municipios con más abstención con respecto al total municipios, mientras que en las regiones Andina y Orinoquia este porcentaje es menor.

A nivel departamental, Casanare, Quindío, Meta, Risaralda y Cundinamarca $^{5}$ fueron los departamentos con más municipios en el nivel más bajo de abstención. Esto demuestra, en cierta medida, que la zona centro-oriental del país concentró el mayor porcentaje de municipios donde la no asistencia a las urnas, en comparación con el resto del país, fue más baja. Con respecto al nivel medio de abstención, el 55\% de los municipios de Sucre, el $44 \%$ de los de Santander y Tolima, y el $51 \%$ de los de Caldas se clasifican en esta categoría. Por su parte, Valle, con el 71\%, y Arauca y Córdoba, con el 43\% de sus municipios, fueron los departamentos que concentraron más municipios en el nivel alto de abstención. Finalmente, los departamentos $^{6}$ con más municipios en el nivel más alto de abstención fueron San Andrés, Vaupés, Guainía, Chocó y Atlántico. Dicha distribución permite identificar particularidades geográficas en relación con la abstención en la zona suroriental y noroccidental del país.

Tabla 1. Porcentaje de municipios por región según los niveles de abstención en la primera vuelta de las elecciones presidenciales de 2014 en Colombia

REGIÓN

\begin{tabular}{|c|c|c|c|c|c|c|c|}
\hline & & AMAZÓNICA & ANDINA & CARIBE & INSULAR & ORINOQUÍA & PACÍFICO \\
\hline \multirow{5}{*}{ Abstención } & Baja & $6,45 \%$ & $29,69 \%$ & $5,64 \%$ & $0 \%$ & $61,82 \%$ & $0 \%$ \\
\hline & Media & $6,45 \%$ & $30,21 \%$ & $17,95 \%$ & $0 \%$ & $10,91 \%$ & $3,33 \%$ \\
\hline & Alta & $19,35 \%$ & $23,78 \%$ & $34,87 \%$ & $0 \%$ & $18,18 \%$ & $20 \%$ \\
\hline & Muy alta & $67,74 \%$ & $16,32 \%$ & $41,54 \%$ & $100 \%$ & $9,09 \%$ & $76,67 \%$ \\
\hline & Total & $100 \%$ & $100 \%$ & $100 \%$ & $100 \%$ & $100 \%$ & $100 \%$ \\
\hline
\end{tabular}

Fuente: Construcción propia a partir de los datos extraídos de la Registraduría Nacional del Estado Civil.

Para comprender de manera sustancial este fenómeno, más allá de su comportamiento regional, es necesario identificar las causas o factores del abstencionismo a la luz de variables macro para entender cuáles son algunos de los determinantes sociodemográficos del abstencionismo. Para el presente estudio se han escogido siete variables ${ }^{7}$, que posteriormente son utilizadas en un modelo de regresión estadístico.
El primer grupo de variables está compuesto por el tamaño de la población ${ }^{8}$ y el Índice de Dimensión Urbana ${ }^{9}$. Su selección obedece a que, según autores como Boix y Riva (2000), Caren (2007), Frandsen (2002) y Funk (2008), el tamaño de la población se relaciona directamente con la abstención - o inversamente con la participación electoral - debido a que en las poblaciones más pequeñas se refuerzan los dispositivos de control

\footnotetext{
${ }^{4}$ Los municipios con porcentajes de abstención menores al 55,3 \% fueron clasificados bajo la categoría de bajo nivel de abstención. Aquellos que se encuentran entre el $55,4 \%$ y el $60,92 \%$ se inscriben en la categoría medio nivel de abstención. Para los que se ubican entre el $60,93 \%$ y el $66,45 \%$ fue asignada la categoría alto nivel de abstención. Finalmente, los municipios que registraron valores mayores a $66,46 \%$ fueron clasificados con la tcategoría muy alto nivel de abstención. La escogencia de estos cuatro grupos responde a la división en cuartiles de los datos recolectados.

517 de los 19 municipios del Casanare, es decir, el $89 \%$, se ubican en un nivel bajo de abstención. Para el caso del Quindío, el $75 \%$ de sus municipios se clasifican bajo esta categoría. En relación con el departamento del Meta, 17 de sus 29 poblaciones, equivalente al $59,9 \%$, registraron niveles bajos de abstención. Por su parte, el $57,1 \%$ de los municipios de Risaralda y Cundinamarca se encuentran clasificados en este grupo.

${ }^{6}$ En los departamentos de San Andrés y el Vaupés, todos los municipios registraron niveles muy altos de abstención. 8 de los 9 municipios de Guainía registraron porcentajes de abstención mayores al 66,46\%. Con respecto a Chocó, 23 de las 30 poblaciones, equivalente al $73 \%$, se ubican en esta categoría. Por su parte, el 73,9\% de las localidades del Atlántico alcanzaron niveles muy altos de abstención.

${ }^{7} \mathrm{La}$ falta de datos agregados que caractericen a los territorios ha constituido un obstáculo para los estudios electorales en América Latina. Bedoya (2015) plantea que aún no se puede considerar un subcampo de estudio, en parte, por la dificultad de acceder a información de manera sistemática.

${ }^{8}$ El tamaño de la población expresa el número de habitantes por municipio (DANE, 2014).

${ }^{9}$ El Índice de Dimensión Urbana asume valores de 0 a 1, donde 0 representa al municipio de Colombia con la menor dinámica urbana y 1 al municipio con la mayor (Departamento Nacional de Planeación, 2014).
} 
social entre sus ciudadanos, desincentivando la abstención. En el segundo grupo de variables se encuentran el Valor Agregado Municipal ${ }^{10}$, los ingresos municipales ${ }^{11}$ y la incidencia de la pobreza según el Índice de la Pobreza Multidimensional ${ }^{12}$. Estos datos permitirán determinar si se cumplen, a nivel municipal, los postulados de la teoría de los recursos, la cual expresa que mientras menos recursos dispongan los ciudadanos, estarán menos dispuestos a votar (Verba y Nei, 1972). En el tercer grupo de variables está la tasa de homicidios ${ }^{13}$ y el indicador de Presencia e Intensidad del Conflicto Armado ${ }^{14}$. Estas variables son fundamentales porque servirán para corroborar si, en efecto, las condiciones de violencia influyen en la abstención, algo especial en el caso colombiano.

Al cruzar la abstención con estas variables, se encuentra que, con respecto al primer grupo, en Antioquia, Arauca, Boyacá, Caldas, Caquetá, Casanare, Cauca, Cundinamarca, Guaviare, Meta, Quindío, Risaralda, Santander y Tolima, la abstención es menor en municipios con mayor población. El análisis denota que estos casos se comportan en contravía de los postulados teóricos, lo que hace necesario considerar otros determinantes para estas elecciones en específico. Por su parte, Atlántico, Bolivar, Chocó, Guaviare, La Guajira, Magdalena, Sucre y Valle demuestran que la abstención aumenta en los municipios donde su población también crece. En el caso de la Dimensión Urbana, los departamentos de Antioquia, Arauca, Boyacá, Caldas, Cauca, Caquetá, Cundinamarca, Meta, Risaralda, Santander y Tolima revelan que disminuye la abstención en la medida en la que sus municipios adquieren una mayor dinámica urbana. Magdalena constituye el único departamento donde la abstención aumentó en razón al incremento de la Dimensión Urbana de sus municipios. En los demás departamentos no se encontraron patrones entre las variables.

Tomando en consideración las variables de corte económico, se interpreta que, en los departamentos de Antioquia, Arauca, Boyacá, Caldas, Cundinamarca, Quindío, Risaralda y
Santander, el abstencionismo es menor en aquellos municipios donde el Valor Agregado Municipal $\mathrm{y}$ los ingresos municipales son mayores. Por su lado, Atlántico, Bolivar, La Guajira, Magdalena y Valle demuestran que la abstención es mayor en los municipios donde se presenta un incremento de estas variables, lo que refleja una contradicción según lo propuesto por la teoría. Quizá la variable que más pistas arroja al respecto es la incidencia de la pobreza según el Índice de la Pobreza Multidimensional, pues es la única de este corte que reflejó regularidad y los resultados esperados al cruzarse con la abstención municipal. Para la mayoría de los departamentos, a excepción de Valle y Vichada, la ausencia de los electores en las urnas es mayor en los municipios con niveles más altos para el índice en cuestión.

Con respecto a las variables relacionadas con la violencia, aunque los patrones no se identifican con tanta fuerza, podría decirse que Antioquia, Atlántico, Caldas, Casanare, Chocó, Córdoba, Guaviare, La Guajira, Norte de Santander, Quindío, Putumayo, Risaralda, Sucre y Vichada se comportaron según lo esperado, puesto que la abstención es mayor en los municipios donde la tasa de homicidios es mayor. Para el caso del indicador de Presencia e Intensidad del Conflicto Armado, los departamentos de Atlántico, Bolivar, Caquetá, Cauca, Magdalena, Meta, Nariño, Putumayo, Santander, Sucre y Valle reflejan que la media de la abstención es mayor en los municipios fuertemente afectados y con conflicto persistente, lo que deja entrever que la violencia puede ser un factor determinante para entender el abstencionismo electoral.

Si bien esta descripción de los datos permite identificar ciertas particularidades o patrones de la abstención con las características de los municipios, es necesario realizar una regresión lineal múltiple con el fin de determinar la relación existente entre la variable dependiente ${ }^{15}$ y las siete variables independientes descritas anteriormente. Antes de mostrar los resultados del modelo, es imprescindible hacer tres aclaraciones metodológicas. La primera

${ }^{10} \mathrm{EI}$ Valor Agregado Municipal corresponde al aporte, en miles de millones de pesos, de cada municipio al PIB departamental (DANE, 2014).

${ }^{11}$ Los ingresos municipales remiten a la suma de los ingresos recibidos por el municipio correspondiente a los ingresos tributarios, las regalías y el

Sistema General de Participaciones (Departamento Nacional de Planeación, 2012).

${ }^{12}$ Esta variable expresa el porcentaje de la incidencia de la pobreza según el Índice de la Pobreza Multidimensional (DANE, 2005).

${ }^{13}$ Tasa de homicidios por cada 100.000 habitantes (Departamento Nacional de Planeación-Sijin, 2012).

${ }^{14} \mathrm{El}$ indicador de Presencia e Intensidad del Conflicto Armado clasifica a los municipios del país de acuerdo con la duración e intensidad del conflicto entre 2000 y 2012. Los valores asignados según las categorías son: 1. Fuertemente afectados y persistente. 2. Levemente afectados y persistente. 3. Fuertemente afectados e interrumpido. 4. Levemente afectados e interrumpido. 5. Levemente afectados y finalizado. 6 . Sin conflicto (Centro de Recursos para el Análisis de Conflictos - CERAC, 2014).

${ }^{15}$ Corresponde al porcentaje de abstención en la primera vuelta de las elecciones presidenciales de 2014 en cada uno de los 1.122 municipios de Colombia. Los datos fueron extraídos del aplicativo digital Con Voto, proporcionado por la Registraduría Nacional del Estado Civil. 
tiene que ver con la calidad de la información, pues, como se dijo anteriormente, la ausencia de datos actualizados impide obtener resultados más precisos. Lo que se busca identificar en el modelo, más que el valor, es la significancia y el signo de la relación entre las variables. En segundo lugar, cinco de las categorías del indicador de Presencia e Intensidad del Conflicto Armado fueron transformadas en variables dummies, dejando por fuera del modelo la categoría Fuertemente afectados y persistente. En tercer lugar, a las variables del tamaño de la población, el Valor Agregado Municipal y los ingresos municipales se les aplicó un logaritmo natural con el fin de equiparar los valores de todas las variables. Los resultados del modelo 1 se presentan en la Tabla 2 .

Tabla 2. Determinantes sociodemográficos del abstencionismo electoral en la primera vuelta de las elecciones presidenciales de 2014 en Colombia. Regresión lineal múltiple

\begin{tabular}{|c|c|c|c|c|}
\hline MODELO 1 & B & $\begin{array}{c}\text { EROR } \\
\text { ESTÁNDAR }\end{array}$ & $\mathbf{t}$ & Sig. \\
\hline (Constante) & 25,455 & 3,980 & 6,396 & 0,000 \\
\hline $\begin{array}{c}\text { Tamaño de la } \\
\text { población }\end{array}$ & 3,124 & 0,511 & 6,114 & 0,000 \\
\hline $\begin{array}{c}\text { Dimensión } \\
\text { urbana }\end{array}$ & 0,038 & 0,037 & 1,004 & 0,316 \\
\hline $\begin{array}{c}\text { Valor } \\
\text { Agregado } \\
\text { Municipal }\end{array}$ & $-1,102$ & 0,389 & $-2,836$ & 0,005 \\
\hline $\begin{array}{c}\text { Ingresos } \\
\text { Municipales }\end{array}$ & -0882 & 0,666 & $-1,324$ & 0,186 \\
\hline $\begin{array}{l}\text { Indice de } \\
\text { Pobreza }\end{array}$ & 0,293 & 0,017 & 17,471 & 0,000 \\
\hline $\begin{array}{l}\text { Homicidios } \\
\text { por cada } \\
100 \text { mil } \\
\text { habitantes }\end{array}$ & 0,041 & 0,006 & 7,374 & 0,000 \\
\hline $\begin{array}{l}\text { Levemente } \\
\text { afectado y } \\
\text { persistente }\end{array}$ & 5,185 & 4,839 & 1,072 & 0,284 \\
\hline $\begin{array}{l}\text { Fuertemente } \\
\text { afectado e } \\
\text { interrumpido }\end{array}$ & -2136 & 1,462 & $-1,462$ & 0,144 \\
\hline $\begin{array}{c}\text { Levemente } \\
\text { afectado e } \\
\text { interrumpido }\end{array}$ & $-3,310$ & 1,182 & $-2,800$ & 0,005 \\
\hline $\begin{array}{l}\text { Levemente } \\
\text { afectado y } \\
\text { finalizado }\end{array}$ & $-2,581$ & $-1,390$ & $-1,856$ & 0,064 \\
\hline Sin comflicto & $-3,428$ & 1,351 & $-2,538$ & 0,011 \\
\hline $\mathbf{R}$ cuadrado & \multicolumn{4}{|c|}{0,392} \\
\hline $\begin{array}{l}\text { R cuadrado } \\
\text { ajustado }\end{array}$ & \multicolumn{4}{|c|}{0,385} \\
\hline $\begin{array}{l}\text { Durbin- } \\
\text { Watson }\end{array}$ & \multicolumn{4}{|c|}{1,586} \\
\hline
\end{tabular}

Fuente: construcción propia
Los resultados del modelo demuestran que el comportamiento de las variables es estadísticamente significativo, a excepción de la Dimensión Urbana, los ingresos municipales y las variables dummies Levemente afectados $y$ persistente y Fuertemente afectados e interrumpido. $\mathrm{Si}$ bien en la descripción inicial de los datos se evidenció que el tamaño de la población se comportaba, de manera diferenciada por departamento, los resultados generales demuestran que esta variable se relaciona directamente con la abstención. Esto puede explicarse partiendo del supuesto de que, en las poblaciones más grandes, a diferencia de las pequeñas, el vínculo entre ciudadanos es menor y, por ende, es posible que los flujos de información, producto de la cercanía y asociación entre los electores, sea menos sustantivo. Así, mientras los ciudadanos estén más dispersos, su disposición a abstenerse será mayor, puesto que su sentido comunitario es más débil y su participación en las actividades que podrían dotarlos de información sobre los candidatos es menor (Boix y Riva, 2000). Además, mientras mayor sea el tamaño de la población, los dispositivos de control son menos efectivos, lo que hace que exista menos presión social para votar, de modo que los ciudadanos perciben que abstenerse no será visto como un comportamiento reprochable por la comunidad (Ben-Bassat y Dahan, 2012; Ortega y Trujillo, 2013; Caren, 2007; Frandsen, 2002; Funk, 2008).

Con respecto a las variables de corte económico, el modelo expresa que el Valor Agregado Municipal y los ingresos municipales se relacionan inversamente con la abstención, es decir, mientras más recursos dispongan los municipios, menor es el porcentaje de abstención. Por su parte, la incidencia de la pobreza, según el Índice de la Pobreza Multidimensional, se relaciona directamente con la abstención, lo que indica que mientras más pobre sean los individuos de un municipio, más posibilidades hay de que sus habitantes no salgan a votar. Esta relación se puede explicar desde la teoría de los recursos, según la cual los individuos con menor capacidad económica, al tener menos dinero, tiempo y capacidades educativas para adquirir información e involucrarse en actividades políticas, se abstienen más que quienes viven más cómodamente (Verba y Nei, 1972).

La variable de Homicidios por cada 100 mil habitantes demostró efectos positivos. Para el caso de las variables dummies significativas, correspondientes al indicador de Presencia e 
Intensidad del Conflicto Armado, el signo negativo de las mismas expresa que, en comparación con los municipios fuertemente afectados y con conflicto persistente, la abstención disminuyó donde la intensidad del conflicto también lo hizo. Es decir, mientras más intenso es el conflicto, mayor es el abstencionismo. ¿Qué mecanismo explicativo se le puede atribuir a los efectos de estas variables de violencia? Como lo plantea Aguilera (2002), las percepciones de inseguridad propias de los contextos de violencia producen en los ciudadanos sentimientos de angustia y miedo, de tal manera que inhiben las conductas y expresiones públicas. Esto conduce a que el comportamiento electoral de los ciudadanos "sea planificado como una medida de defensa y autoprotección frente al contexto que se percibe como amenazante" (Aguilar, 2002, p.11). En ese sentido, la violencia se perfila como un determinante de la abstención en cuanto que modifica las prácticas sociales y políticas de los ciudadanos, los cuales evitan exponerse en lo público y, por ende, se sienten limitados para asistir a las urnas ${ }^{16}$.

\section{Explicaciones micro del abstencionismo electoral}

Los resultados encontrados en el apartado anterior demuestran que el abstencionismo electoral, para la primera vuelta de las elecciones presidenciales de 2014, puede ser explicado por factores sociodemográficos como el tamaño de la población, la capacidad económica de los municipios y la violencia. Si bien este tipo de análisis de datos agregados ${ }^{17}$ advierte características generales y pautas relevantes en el comportamiento electoral a nivel municipal, es evidente que este fenómeno no puede ser abordado únicamente por esta vía, pues omite la incidencia de las actitudes y las preferencias individuales, la cuales son fundamentales en la decisión del elector.

En cuanto a las percepciones y representaciones que se gestan en los electores, es posible identificar un lugar común en los estudios sobre la abstención. Como bien se dijo anteriormente, la abstención puede ser entendida como la respuesta activa y consciente de una parte de la ciudadanía que, debido a los sentimientos de ineficacia, cinismo y desconfianza que ostentan hacia las instituciones y la democracia, deciden no asistir a las urnas el día de las elecciones (Mikhail y Cortéz, 2014; Salazar y Temkin, 2007 y Millán, 2013). Esto quiere decir que la no participación es asumida como una conducta voluntaria que obedece a la desafección política que los individuos manifiestan contra instituciones de la democracia como los partidos, el poder ejecutivo y legislativo, e incluso las elecciones mismas. Es por esto que resulta imprescindible acudir a los datos individuales proporcionados por las encuestas, con el fin de abordar esta dimensión del abstencionismo electoral y completar así el panorama de análisis.

Teniendo en cuenta lo anterior, se usan los datos de la encuesta del Barómetro de las AméricasLapop del año 2016, asumiendo como variable dependiente la respuesta No votó a la pregunta “¿Votó usted en las últimas elecciones presidenciales de 2014?". Como variables independientes que dan cuenta de la desafección política de los ciudadanos, se escogieron las preguntas por la satisfacción con la democracia y la confianza en los partidos políticos, el presidente y las elecciones. Además, se incluyen las variables de tamaño de la población e ingresos mensuales de los individuos, para así corroborar a nivel individual los resultados encontrados en el modelo de datos agregados.

Al igual que en el apartado anterior, antes de mostrar los resultados de la regresión, es necesario describir el comportamiento de la variable dependiente al ser cruzada con cada una de las variables independientes. Las pruebas de Chi-cuadrado ${ }^{18}$ arrojaron valores menores a 0,05 para todas las variables, lo que demuestra que son significativas, a excepción de la satisfacción con la democracia, de tal manera que queda descartada.

Para el caso del tamaño de la población, los datos demuestran que se abstuvieron más los habitantes de ciudades capitales que los pobladores de ciudades pequeñas o zonas rurales, coincidiendo con los resultados antes explicados. También se evidencia la tendencia a abstenerse de votar de las personas con menores ingresos, lo que deja entrever que a nivel individual también se pueden cumplir los postulados de la teoría de los recursos (ver Tabla 4). En relación con las variables vinculadas

\footnotetext{
${ }^{16}$ En este punto podría objetarse que comicios que estuvieron permeados por contextos más violentos, como las elecciones presidenciales de 2002 y 2006, tuvieron niveles más bajos de abstención. A esto se puede responder que los efectos de la violencia sobre los electores son más estructurales que contextuales, lo que significa que, aunque las elecciones de 2014 presenten condiciones de seguridad más favorables que las de años anteriores, las secuelas sobre los individuos perviven y, por ende, inciden en su comportamiento electoral.

${ }^{17}$ Es necesario mencionar que el uso de datos agregados puede traer consigo la problemática de las falacias ecológicas, las cuales consisten en interpretar fenómenos que obedecen a decisiones individuales desde las características de los agregados.

${ }^{18} \mathrm{El}$ Chi-cuadrado del tamaño de la población, la confianza en el presidente y las elecciones fue de 0 . El valor de la confianza en los partidos fue de 0,006, mientras que el de los ingresos personales fue de 0,011. El Chi-cuadrado de la satisfacción con la democracia fue de 0,590.
} 
con la desafección política, se puede identificar que la abstención es mayor en los ciudadanos con mayor desconfianza en los partidos, el presidente y las elecciones, de tal manera que estos factores psicológicos pueden incidir en la ausencia de los electores en las urnas (ver Tablas 5, 6 y 7).

Tabla 3. Porcentaje de personas que declararon votar o abstenerse en la primera vuelta de las elecciones presidenciales de 2014 según el tamaño de la población al que pertenece.

\begin{tabular}{cccc} 
& & \multicolumn{2}{c}{$\begin{array}{c}\text { Voto en la primera } \\
\text { vuelta de las elecciones } \\
\text { presidenciales de 2014 }\end{array}$} \\
\cline { 2 - 4 } & & Votó & No votó \\
\hline \multirow{2}{*}{ Tamaño } & $\begin{array}{l}\text { Ciudad } \\
\text { capital }\end{array}$ & $51,9 \%$ & $48,1 \%$ \\
\cline { 2 - 4 } población & $\begin{array}{l}\text { Ciudad } \\
\text { grande }\end{array}$ & $59,3 \%$ & $40,7 \%$ \\
\cline { 2 - 4 } & $\begin{array}{l}\text { Ciudad } \\
\text { media }\end{array}$ & $66,5 \%$ & $33,5 \%$ \\
\cline { 2 - 4 } & $\begin{array}{c}\text { Ciudad } \\
\text { pequeña }\end{array}$ & $70,3 \%$ & $29,7 \%$ \\
\cline { 2 - 4 } & $\begin{array}{l}\text { Zona } \\
\text { rural }\end{array}$ & $68,7 \%$ & $31,3 \%$ \\
\hline
\end{tabular}

Fuente: construcción propia a partir de los resultados de la encuesta Baróm

Tabla 5. Porcentaje de personas que declararon votaro abstenerse en la primera vuelta de las elecciones presidenciales de 2014 según el nivel de confianza en los partidos políticos.

\begin{tabular}{cccc} 
& & \multicolumn{2}{c}{$\begin{array}{c}\text { Voto en la primera } \\
\text { vuelta de las elecciones } \\
\text { presidenciales de 2014 }\end{array}$} \\
\cline { 2 - 4 } & & Votó & No votó \\
\cline { 2 - 4 } & Nada & $55,4 \%$ & $44,6 \%$ \\
\cline { 2 - 4 } $\begin{array}{c}\text { en los } \\
\text { partidos } \\
\text { politicos }\end{array}$ & $\mathbf{2}$ & $67,2 \%$ & $32,8 \%$ \\
\cline { 2 - 4 } & $\mathbf{3}$ & $61,9 \%$ & $38,1 \%$ \\
& $\mathbf{5}$ & $63,1 \%$ & $36,9 \%$ \\
\cline { 2 - 4 } & $\mathbf{6}$ & $67,0 \%$ & $33,0 \%$ \\
\cline { 2 - 4 } & & $69,4 \%$ & $30,6 \%$ \\
\cline { 2 - 4 } & $\mathbf{4}$ & $75,0 \%$ & $25,0 \%$
\end{tabular}

Fuente: construcción propia a partir de los resultados de la encuesta Barómetro de las Américas-Lapop 2016.
Tabla 4. Porcentaje de personas que declararon votaro abstenerse en la primera vuelta de las elecciones presidenciales de 2014 según el ingreso personal mensual.

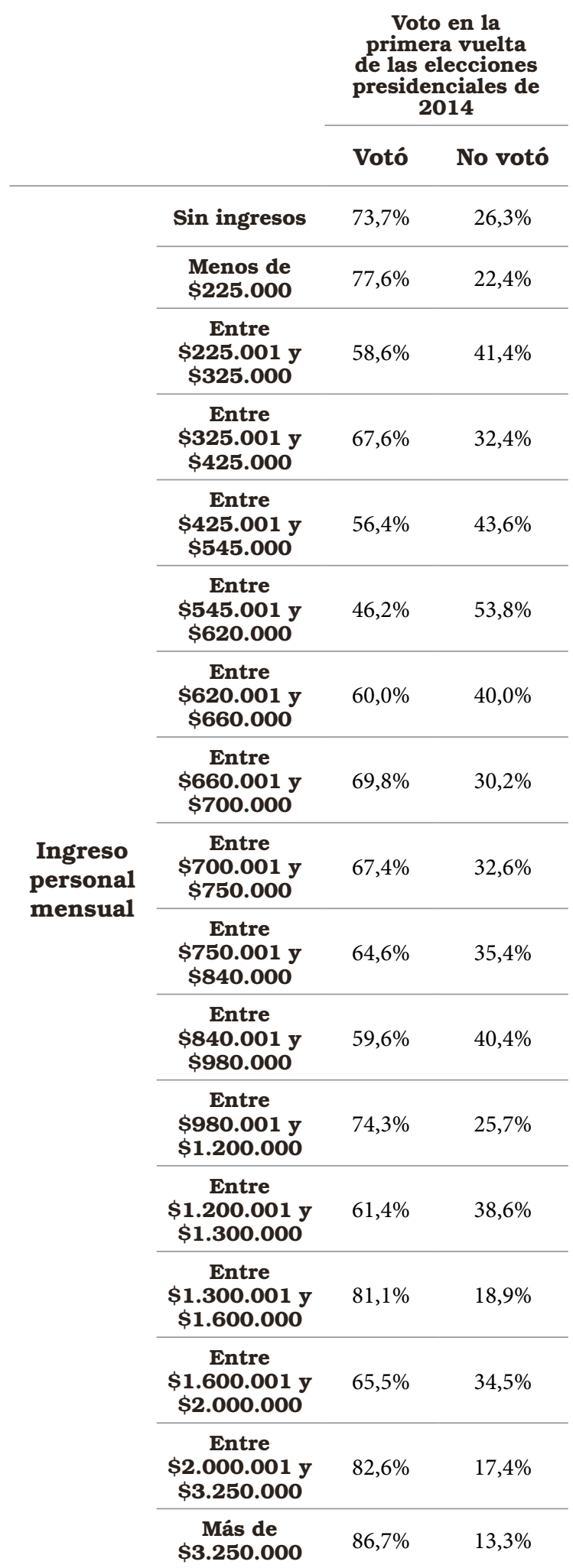

Fuente: construcción propia a partir de los resultados de la encuesta Barómetro de las Américas-Lapop 2016. 
Tabla 6. Porcentaje de personas que declararon votar o abstenerse en la primera vuelta de las elecciones presidenciales de 2014 según el nivel de confianza en el Presidente.

\begin{tabular}{cccc} 
& & \multicolumn{2}{c}{$\begin{array}{c}\text { Voto en la primera } \\
\text { vuelta de las elecciones } \\
\text { presidenciales de 2014 }\end{array}$} \\
\cline { 2 - 4 } & & Votó & No votó \\
\hline & Nada & $53,3 \%$ & $46,7 \%$ \\
\cline { 2 - 4 } & $\mathbf{2}$ & $61,9 \%$ & $38,1 \%$ \\
Confianza & $\mathbf{3}$ & $65,2 \%$ & $34,8 \%$ \\
\cline { 2 - 4 } en el & $\mathbf{4}$ & $60,5 \%$ & $39,5 \%$ \\
\cline { 2 - 4 } presidente & $\mathbf{5}$ & $67,2 \%$ & $32,8 \%$ \\
\cline { 2 - 4 } & $\mathbf{6}$ & $77,2 \%$ & $22,8 \%$ \\
\cline { 2 - 4 } & $\mathbf{M u c h a}$ & $72,1 \%$ & $27,9 \%$
\end{tabular}

Fuente: construcción propia a partir de los resultados de la encuesta Barómetro de las Américas-Lapop 2016.
Tabla 7. Porcentaje de personas que declararon votaro abstenerse en la primera vuelta de las elecciones presidenciales de 2014 según el nivel de confianza en las eleccionesa.

\begin{tabular}{cccc} 
& & \multicolumn{2}{c}{$\begin{array}{c}\text { Voto en la primera } \\
\text { vuelta de las elecciones } \\
\text { presidenciales de 2014 }\end{array}$} \\
\cline { 2 - 4 } & & Votó & No votó \\
\hline & Nada & $52,0 \%$ & $48,0 \%$ \\
\cline { 2 - 4 } & $\mathbf{2}$ & $57,1 \%$ & $42,9 \%$ \\
Confianza & $\mathbf{3}$ & $59,9 \%$ & $40.1 \%$ \\
\cline { 2 - 4 } en las & $\mathbf{4}$ & $62,3 \%$ & $37,7 \%$ \\
\cline { 2 - 4 } elecciones & $\mathbf{5}$ & $70,9 \%$ & $29,1 \%$ \\
\cline { 2 - 4 } & $\mathbf{6}$ & $77,9 \%$ & $22,1 \%$ \\
\cline { 2 - 4 } & $\mathbf{M u c h a}$ & $75,4 \%$ & $24,6 \%$
\end{tabular}

Fuente: construcción propia a partir de los resultados de la encuesta Barómetro de las Américas-Lapop 2016.

Ahora bien, para identificar la relación estadística entre las variables, se realiza una regresión logística binominal. Todas las variables fueron recodificadas en variables dummies. Así, la confianza en los partidos políticos, el presidente y las elecciones se transformaron en las variables Poca confianza ${ }^{19}$, Media confianza ${ }^{20}$ y Mucha confianza ${ }^{21}$. En el caso de los ingresos, se crearon las variables Menos de $\$ 545.000$ pesos, Entre $\$ 545.001$ y $\$ 750.000$ pesos, Entre $\$ 750.001$ y \$1’300.000 pesos y Más de \$1’300.001 pesos. Por su parte, el tamaño de la población se convirtió en las variables Ciudad capital, Ciudad grande, Ciudad mediana, Ciudad pequeña y Zona rural. Los resultados del modelo 2 se presentan en la Tabla 8.

Tabla 8. Determinantes del abstencionismo electoral en la primera vuelta de elecciones presidenciales de 2014 en Colombia. Regresión logística binominal2 ${ }^{22}$

No votó en la primera vuelta de las elecciones

Modelo 2 presidenciales de 2014

\begin{tabular}{|c|c|c|c|c|c|c|}
\hline \multirow{2}{*}{ Modelo 2} & \\
\hline & B & $\begin{array}{c}\text { Error } \\
\text { estándar }\end{array}$ & Wald & g1 & Sig. & $\operatorname{Exp}(B)$ \\
\hline Ciudades grandes & $-0,371$ & 0,206 & 3,251 & 1 & 0,071 & 0,690 \\
\hline Ciudades medianas & $-0,875$ & 0,335 & 6,820 & 1 & 0,009 & 0,417 \\
\hline Ciudades pequeñas & $-0,710$ & 0,408 & 3,034 & 1 & 0,082 & 0,492 \\
\hline Zona rural & $-0,817$ & 0,263 & 9,639 & 1 & 0,002 & 0,442 \\
\hline Confianza media en los partidos politicos & $-0,020$ & 0,195 & 0,011 & 1 & 0,916 & 0,980 \\
\hline Mucha confianza en los partidos politicos & $-0,163$ & 0,553 & 0,087 & 1 & 0,768 & 0,849 \\
\hline Confiamza media en el ejecutivo & $-0,323$ & 0,193 & 2,817 & 1 & 0,093 & 0,724 \\
\hline Mucha confianza en el ejecutivo & $-0,439$ & 0,308 & 2,024 & 1 & 0,155 & 0,645 \\
\hline Confianza media en las elecciones & $-0,237$ & 0,183 & 1,667 & 1 & 0,197 & 0,789 \\
\hline Mucha confianza en las elecciones & $-0,580$ & 0,347 & 2,790 & 1 & 0,095 & 0,560 \\
\hline Ingresos entre $\$ 545.001$ y $\$ 750.000$ & $-0,219$ & 0,216 & 1,021 & 1 & 0,312 & 1,244 \\
\hline Ingresos entre $\$ 750.001$ y $\$ 1.300 .000$ & $-0,122$ & 0,207 & 0,347 & 1 & 0,556 & 0,885 \\
\hline Ingresos de más de $\$ 1.300 .000$ pesos & $-0,762$ & 0,287 & 7,034 & 1 & 0,008 & 0,467 \\
\hline Constante & 0,198 & 0,249 & 0,636 & 1 & 0,425 & 1,219 \\
\hline
\end{tabular}

Fuente: construcción propia.

\footnotetext{
${ }^{19}$ En esta categoría se agrupan las respuestas con valores correspondientes a 1 y 2.

${ }^{20}$ En esta categoría se agrupan las respuestas con valores correspondientes a 3,4 y 5 .

${ }^{21}$ En esta categoría se agrupan las respuestas con valores correspondientes a 6 y 7.

${ }^{22}$ La variable dependiente es 0 si el encuestado declara haber votado en la primera vuelta presidencial de 2014 y 1 caso contrario.
} 
Leyendo los resultados de este ejercicio a un nivel de significancia del $10 \%$, las variables correspondientes al tamaño de la población expresan que, en comparación con las ciudades capitales, el abstencionismo aumenta en la medida en que también lo hace el tamaño de la población. Es decir, mientras más grandes son las ciudades, más se abstienen los ciudadanos. Estos resultados a nivel individual refuerzan los hallazgos encontrados en el análisis agregado de los datos, lo que demuestra que el tamaño de la población, al relacionarse con asuntos como el apego en comunidad y la presión social, incide en el comportamiento electoral.

Para el caso de los ingresos individuales, solo la variable de Ingresos de más de \$1’300.000 pesos es significativa. El signo negativo del coeficiente indica que, a mayores ingresos, menor abstención. Esto significa que, a pesar de que las otras variables no permiten entablar una relación más significativa para corroborar lo expuesto en el apartado anterior, el ingreso se perfila como un determinante relevante que permite explicar la abstención, considerando que mientras más recursos posean los individuos, serán menos proclives a abstenerse.

En cuanto a las variables de corte actitudinal, los resultados revelan que la incidencia de la confianza hacia cada una de las instituciones incide sobre el abstencionismo de manera diferenciada. En la confianza en los partidos políticos, la ausencia de significancia en sus valores indica que no se puede establecer una relación estadística entre la abstención electoral y dicha variable. Esto se puede entender teniendo en consideración el carácter personalista de los comicios presidenciales en Colombia. La existencia de líderes y caudillos que se sobreponen a los partidos políticos configura el comportamiento de los electores, que más que guiarse por los principios o valores que encarnan las organizaciones políticas, se movilizan en virtud de los referentes que enarbolan los personalismos ${ }^{23}$. En ese sentido, los partidos políticos y la confianza que tengan hacia ellos parecen ser menos relevantes en la decisión que toman los ciudadanos en las elecciones presidenciales.

Acerca de la confianza en el ejecutivo, solo la variable Confianza media es significativa y su signo negativo expresa que los individuos con confianza mediaseabstienenmenos en comparación conaquellas personas que tienen poca confianza. Cabe aclarar que su relación estadística con el abstencionismo no es del todo determinante, pues es posible que el elector, más que votar retrospectivamente, lo haga prospectivamente (Fiorina, 1978); es decir, pensando en la capacidad de los candidatos de enfrentar retos futuros.

Con respecto a la confianza en las elecciones, únicamente la variable Mucha confianza en las elecciones es significativa. Al igual que en el caso anterior, el signo negativo del coeficiente indica que los individuos con mucha confianza se abstienen menos en comparación con los individuos que tienen poca confianza en las elecciones.

En definitiva, los resultados del modelo de regresión logística demuestran que aun cuando la confianza en estas instituciones no se constituye como una variable decisiva o determinante para explicar la abstención electoral en la primera vuelta de las elecciones presidenciales de 2014, sí se configura, junto a otros factores, como uno de los causantes de la ausencia de los electores en las urnas. Cuando la ciudadanía ostenta sentimientos de lejanía o desconfianza hacia las instituciones políticas y carece de referentes de identificación, es poco probable que su comportamiento se traduzca en actos de participación política, como es el ejercicio de votar. En ese sentido, la abstención se entiende como un acto voluntario y activo que expresa la desafección política de los individuos, que inconformes con el accionar de los poderes públicos y convencidos de que los representantes no actúan en virtud de los intereses de la ciudadanía, prefieren abstenerse antes de alzar su voz y manifestar su inconformidad por medio del voto.

\section{Conclusión}

En el estudio del comportamiento electoral ha sido recurrente preguntarse por las causas de la participación de los ciudadanos en los comicios. Un fenómeno que no ha recibido la misma atención dentro del subcampo de estudio del abstencionismo, el cual trae consigo serios cuestionamientos a los procesos democráticos, pues la ausencia de electores en las urnas socava la legitimidad de los elegidos.

El caso colombiano se presenta como idóneo para este tipo de estudios, ya que la participación electoral ha sido tradicionalmente

\footnotetext{
${ }^{23}$ Para Manin (1998), las democracias de audiencias traen consigo la personalización del poder, particularmente en aquellos países donde el poder ejecutivo es elegido directamente. Esto quiere decir que, si bien los partidos políticos no desaparecen, su función como referentes ideológicos pierde relevancia, ya que se ven obligados a convertirse en instrumentos que están a disposición de los personalismos.
} 
baja. Los porcentajes de abstención en los últimos 20 años oscilan entre el $60 \%$ y el $40 \%$. De allí que el abstencionismo se erija como fenómeno que es necesario abordar desde la ciencia política. En este artículo se identificaron algunos factores $-\mathrm{y}$ los mecanismos explicativos según lo que propone la literatura- que explican el abstencionismo en la primera vuelta de las elecciones presidenciales de $2014^{24}$.

El análisis agregado de los datos permitió reconocer que ciertas características sociodemográficas de los municipios tienen efectos sobre la abstención. La relación positiva entre el tamaño de la población y el abstencionismo demuestra que la cercanía entre los individuos, el apego hacia la comunidad y la presión social pueden incidir en el comportamiento electoral.

Las variables de corte económico señalan que los recursos a nivel municipal contribuyen a explicar el objeto de estudio, en el sentido de que menores niveles agregados de riqueza se traducen en mayor abstención. La relación positiva entre la incidencia de pobreza según el Índice Multidimensional de Pobreza y la ausencia de los electores confirma lo anterior. Hay que repetir que estas variables están vinculadas por el mecanismo explicativo propuesto por autores como Verba y Nie (1972), los cuales afirman que mientras más recursos tengan los individuos, tendrán más tiempo y habilidades educativas para informarse sobre los procesos electorales.

Quizá el hallazgo más novedoso presentado en este estudio tiene que ver con la relación que se establece entre la violencia y el abstencionismo electoral. Tanto la tasa de homicidios como el indicador de Presencia e Intensidad del Conflicto Armado expresan que la abstención es menor donde se presentaron menos asesinatos y donde el conflicto ha sido menos intenso. Estos resultados resaltan la importancia de tener en consideración variables como la violencia, que adquieren importancia explicativa, al menos en contextos tan convulsionados como los propios de América Latina.

Por otro lado, el análisis de los datos extraídos de la encuesta del Barómetro de las Américas-Lapop 2016 permitió corroborar que, a nivel individual, los hallazgos encontrados, en relación con el tamaño de la población y los ingresos, van en la misma dirección que los resultados explicados a nivel agregado. Además, se comprobó que, aunque la confianza en instituciones como los partidos políticos, el presidente y las elecciones no constituye una variable decisiva al momento de explicar la abstención, sí tienen efectos sobre la misma. Esto significa que, si bien el abstencionismo se puede explicar como una respuesta por parte de los ciudadanos que sienten desafección con el sistema, este no es el único causante, sino involucra factores de diversa índole, como pueden ser las características sociodemográficas, los arreglos institucionales, la cultura política, entre otros.

Aunque los resultados encontrados en este estudio solo aplican para las elecciones del 2014 y no pueden ser generalizables, este trabajo sí contribuye a nutrir el campo de los estudios electorales en Colombia, el cual debe tratar con mayor insistencia el abstencionismo como fenómeno característico del comportamiento del elector. Una agenda investigativa en América Latina alrededor de la abstención no solo debería centrarse en realizar estudios comparativos que permitan reconocer regularidades entre regiones o países, sino también enfocarse en referentes más cercanos al elector, como son los sentimientos o percepciones que asumen los ciudadanos sobre el proceso electoral y las subjetividades que estos encarnan con relación a los cargos a elegir.

Finalmente, es importante mencionar que los próximos estudios deben enfocar sus esfuerzos en realizar análisis temporales y entre diferentes niveles de elección, para entender de manera comparativa la variabilidad existente entre las tasas de abstención en un territorio determinado. Para el caso colombiano, esto se hace prioritario a la hora de comprender la incidencia, en el comportamiento electoral, de otros factores como el clientelismo y los delitos electorales, según su presencia regional.

\section{Referencias}

Adams, J., Dow, J. y Merril, S. (2006). The Political Consequences of Alienation-Bases and Indifference-Based Voter Abstentation: Applications to Presidental Elections. Political Behavior, 28 (1), 65-86.

Andersen, J., Fiva, J. y Natavik, G. (2014). Voting When the Stakes Are High. CAMP Working Papers Series, 8, 1-41.

${ }^{24}$ Cabe aclarar que en este estudio no se tomaron en consideración factores propios del abstencionismo técnico, los cuales refieren a determinantes ajenos a la voluntad del individuo, como el clima, los defectos del censo electoral o el estado de salud. 
Aguilera, A. y Fernández, L. (2002). Violencia y elecciones en Santander. Reflexión Política, 8,192-199.

Aguilera, A. (2002). Implicaciones de la violencia política en la intención de voto y en el comportamiento electoral. Reflexión Política, 7, 2-13.

Alcubilla, E. (1998). Abstencionismo electoral. En Diccionario electoral (pp.1-6). San José: Centro Interamericano de Asesorías y Promoción Electoral (CAPEL).

Araya, C. (2014). Análisis estadístico del abstencionismo en las elecciones presidenciales de Costa Rica 2014. Ciencias Económicas, 33 (1), 75-87

Bedoya, J. (2015). Los estudios del comportamiento del elector en América Latina: un análisis bibliográfico de los casos de México, Argentina, Chile y Colombia, 2000-2010. Estudios Politicos, 46, 197-219.

Ben-Bassat, A. y Dahan, M. (2012). Social Identity and Voting Behavior. Public Choice, 151, 193-214.

Bellettini, G., Berti, C y Monfardini, C. (2014). Socio-Economic Heterogenety and Electoral Turnout: An Aggregate Analysis with Precint-Level Data. Working Paper 4999, 1-26.

Boix, C. y Riba, C. (2000). Las bases sociales y políticas de la abstención en las elecciones generales españolas: recursos individuales, movilización estratégica e instituciones. Reis. Revista Española de Investigaciones Sociológicas, 90, 95-128.

Boulding, C. y Brown, D. (2013). Do Political Parties Matter for Turnout? Number of Parties, Electoral Rules and Local Elections in Brazil and Bolivia. Party Politics, 21 (3), 404-416.

Boyd, R. (1986). Elections Calendars and Voter Turnout. American Politics Quarterly, 14 (1-2), 89-104.

Breux, S., Couture, J. y Goodman, N. (2016). Fewer Voters, Higher Stakes? The Applicability of Rational Choice for Voter Turnout. Environment and Planning, Government and Policy, 1-20.

Caicedo, J. (2014). Abstención electoral y voto en blanco en la consolidación democrática colombiana. En Elecciones en Colombia, 2014 ¿Representaciones fragmentadas? (pp.45-85). Bogotá: Fundación Konrad Adenauer.

Caren, N. (2007). Big City, Big Turnout? Electoral Participation in American Cities. Journal of Urban Affairs, 29 (1), 31-46.

Centro de Recursos para el Análisis de Conflictos. (2014). Indicador de presencia e intensidad del conflicto armado en Colombia. Recuperado de: https://docs. google.com/spreadsheets/d/1ITvbEU79Ok_GhaDmN12mCeHBFfBzoa4GfNT68wyAnXo/edit\#gid=0

Correa, A. y Forero, D. (2014). Incentivos al abstencionismo electoral por apatía en ciudadanos bogotanos que nunca han votado. Suma Neg, 5 (12), 105114.
Delgado, I. (1998). El comportamiento electoral en los municipios rurales: una aproximación desde las elecciones municipales de 1995. Agricultura y Sociedad, 86, 13-32.

Departamento Administrativo Nacional de Estadística. (2014). Población municipal. Recuperado de: https://www.dnp.gov.co/programas/desarrollo-territorial/Estudios-Territoriales/Estudios-y-Ejercicios/Paginas/ Tipologias.aspx

Departamento Nacional de Planeación. (2014). Índice de Dimensión Urbana. Recuperado de: https:// www.dnp.gov.co/programas/desarrollo-territorial/Estudios-Territoriales/Estudios-y-Ejercicios/Paginas/Tipologias.aspx

Downs, A. (1957). An economic Theory of Democracy. Nueva York: Harper and Row.

Fiorina, M. (1978). Económic Restropective Voting in América National Elections: A Micro-Análisis. América Journal of Politica Science, 2(22), 426443.

Freitag, M. (2010). Structure versus Culture: A Comparative Study of the Influence of Political Institutions and Cultural Modernization Factor on Voter Turnout in Swiss Sub-national Parlamentary Elections. International Political Science Review, 31 (4), 428-448.

Fornos, C., Power, T. y Garand, J. (2004). Explaining Voter Turnout in Latin America. Comparative Political Studies, 37 (8), 909-940.

Funk, P. (2008). Social Incentives and Voter Turnout: Evidence from the Swiss Mail Ballot System. Journal of the European Economic Association, 8 (5), 1077-1103.

Frandsen, A. (2002). Size and Electoral Participation in Local Elections. Environment and Planning C: Government and Policy, 20, 853-869.

Geys, B. (2006). Explaining voter turnout: A review of aggregate-level research. Electoral Studies, 25, 637-663.

Giraldo, C. (2012). Abstencionismo electoral en las elecciones locales en Medellín. (Tesis de maestría). Instituto de Estudios Políticos. Universidad de Antioquia.

González, J. (1989). La sociología de la abstención. Revista Facultad de Derecho y Ciencias Políticas, 85, 113-121.

Hajnal, Z. y Lewis, P. (2003). Municipal Institutions and Voter Turnout in Local Elections. Urban Affairs Review, 38 (5), 645-668.

Henderson, A. y McEwen, N. (2010). Comparative Analysis of Voter Turnout in Regional Elections. Electoral Studies, 29 (3), 405-416.

Hernández, O. (1990) Análisis del abstencionismo en las elecciones presidenciales de Costa Rica en el período 1953-1986. Anuario de Estudios Cen- 
troamericanos, 16 (2), 117-130.

Hirschman, A. (1977). Salida, voz y lealtad. México: Fondo de Cultura Económica.

Horbath, J. (2004). Pobreza y elecciones en Colombia: algunos hallazgos para reflexionar. Espiral, 10 (29), 199-235.

Hoyos, D. (2009). Dinámicas político-electorales en zonas de influencia paramilitar. Análisis de la competencia electoral y la participación electoral. Análisis Político, 6, 13-32.

Jackman, R. y Miller, R. (1995). Voter Turnout in the Industrial Democracies during the 1980s. Comparative Political Studies, 27 (4), 467-492.

Kaniovski, S. y Mueller, D. (2006). Community Size, Heterogeneity and Voter Turnouts. Public Choice, 12, 399-415.

Kuenzi, M. y Lambrihgt, G. (2007). Voter Turnout in Africa's Multiparty Regimes. Comparative Political Studies, 40 (6), 665-690.

Lago, I., Bermúdez, S., Guinjoan, M. y Simón, P. (2014). Turnout and Fractionalization. GEN Working Paper. Universidad de Vigo, 1-31.

Lefevere, J. y Van Aelst, P. (2014). First-order, Second-order or Third-rate? A Comparison of Turnout in European, Local and National Elections in the Netherlands. Electoral Studies, 35, 159-170.

Lutz, B. (2005). La participación electoral inconclusa: abstencionismo y votación nula en México. Revista Mexicana de Sociología, 67 (4), 796-825.

Manin, B. (1998). Los principios del gobierno representativo. Madrid: Alianza Editorial.

Mijares, F. (2006). "Desafección Política”; principal causa del abstencionismo electoral en México. Revista del Instituto Electoral del Estado de México. Apuntes Electorales, 5 (23), 9-70.

Millán, H. (2013). México: el abstencionismo en democracias incipientes. Ciencia Política, 8 (15), 67106.

Medina, D. (2004). La abstención en las elecciones venezolanas de 1998: recursos y compromisos políticos. Politeia, 32-33, 61-94.

Nohlen, D. (2004). La Participación electoral como objeto de estudio. Elecciones, 3, 137-156).

Ortega, C. y Trujillo, J. (2013). La influencia del tamaño de las localidades sobre la participación electoral: un análisis contextual de las elecciones locales de 2011 en Andalucía. Revista de Investigaciones Politicas y Sociológicas, 12 (1), 71-91.

Plane, D. y Gershtenson, J. (2004). Ideological Locations, Abstention, and Turnout in U.S. Midterm Senate Elections. Political Behavior, 1 (26), 69-93.

Power, T. y Roberts, J. (1995). Compulsory Voting, Invalid Ballots, and Abstention in Brazil. Political Research Buarterly, 4 (48), 795-826.
Registraduria Nacional del Estado Civil. (2014). Resultados electorales 2014.

Registraduría Nacional del Estado Civil; Centro de Estudios en Democracia y Asuntos Electorales (CEDAE); Escuela de Política y Relaciones Internacionales - Grupo de Análisis Político (GAP). (2013). Abstencionismo electoral en Colombia: una aproximación a sus causas. Bogotá: Fondo de Publicaciones de la Universidad Sergio Arboleda.

Riera, P. (2012). La abstención diferencial en la España de las autonomías: pautas significativas y mecanismos explicativos. Revista Internacional de Sociología, 70 (3), 615-642.

Salazar, R. y Temkin, B. (2007). Abstencionismo, escolaridad y confianza en las instituciones Las elecciones federales de 2003 en México. Politica y Gobierno, 14 (1), 5-42.

Seccionales de Investigación Criminal (SIJIN). (2012). Tasa de homicidios por 100 mil habitantes. Recuperado de: https://www.dnp.gov.co/programas/desarrollo-territorial/Estudios-Territoriales/Estudios-y-Ejercicios/Paginas/Tipologias.aspx

Sørensen, R. (2016). The Impact of State Television on Voter Turnout. British Journal of Political Science, 1-22.

Torcal, M. (2006). Desafección institucional e historia democrática en las nuevas democracias. Revista SAAP, 3 (2), 591-634.

Uribe, M. (1995). El malestar de la representación política de la Colombia de hoy. Estudios Políticos, 6, 11-26.

Verba, S. y Nei, N. (1972). Participation in America. Political Democracy and Social Inequality. Nueva York: Harper and Row.

Zavala, I. (2005). Factores sociales de la abstención electoral en las elecciones federales de 2003. Estudios Políticos, 8 (4), 141-155.

Zavala, I. (2010). La abstención electoral como protesta. Estudios Politicos, 9 (21), 113-139.

Zazueta, S., Mikhail, I. y Cortez, W. (2014) Determinantes de la participación electoral en México. Estudios Sociológicos, 32 (95), 323-353. 